\title{
Reasons why customers will still buy premium (chassis) in the age of automated driving
}

Armin Schöpfel

AUDI AG

This manuscript is not available according to publishing restriction.

Thank you for your understanding. 